Research Symposium

\title{
The Inaugural IJSPT and OSET Research Symposium: 2021 Abstracts
}

\author{
International Journal of Sports Physical Therapy ${ }^{1}{ }^{a}$ \\ ${ }^{1}$ North American Sports Medicine Institute \\ Keywords: orthopedic research, sports medicine \\ https://doi.org/10.26603/001c.29925
}

\section{International Journal of Sports Physical Therapy}

Vol. 16, Issue 6, 2021

\begin{abstract}
The International Journal of Sports Physical Therapy is pleased to publish abstracts from the 11th Orthopaedic Summit (OSET) taking place in Las Vegas, December 11-14, 2021. The IJSPT hosted the first annual research forum and reception at OSET, sponsored by ATI Physical Therapy and Hyperice.

The abstracts presented in the following pages were selected by the OSET Research Committee and editorial staff of the International Journal of Sports Physical Therapy. After careful review, a total of 22 research abstracts were accepted and presented at OSET 2021. Awards for outstanding abstracts were presented on December 11 th.

The 2021 abstracts include contemporary orthopaedic and rehabilitation topics across various research designs. Each abstract presents only a brief summary of a research project / presentation and does not permit full assessment of the scientific rigor with which the work was conducted. While the abstracts offer only preliminary results that may require further refinement and future validation, they do serve an important role of sharing new research ideas and rehabilitation advancements. This sharing of ideas helps to encourage dialogue among researchers, clinicians, and educators that will ultimately contribute to the orthopaedic and rehabilitation body of knowledge. We strongly encourage authors to continue pursuing publication of their research as a full manuscript. Thank you to all submitting abstracts for consideration. We look forward to another outstanding season of submissions for OSET 2022.

Phil Page PhD, PT, ATC

Chuck Thigpen PhD, PT, ATC

OSET Research Committee Co-Chairs
\end{abstract}

\section{THE EFFECT OF STRENGTH OR ENDURANCE TRAINING PROGRAMS ON STRENGTH AND ENDURANCE MEASURES OF THE SUPRASPINATUS AND INFRASPINATUS}

\author{
${ }^{1}$ Adair $K,{ }^{1}$ Bauer A, ${ }^{1}$ Kerns $G,{ }^{1}$ Roman $G,{ }^{1}$ Sones $S,{ }^{2}$ Porter $D$, \\ ${ }^{1}$ Smith $B,{ }^{1}$ Manske $R$ \\ ${ }^{1}$ Wichita State University Department of Physical Ther- \\ apy; ${ }^{2}$ Ascension Via Christi Sports Medicine and Family \\ Practice
}

Background: Shoulder pain is the third most common cause of musculoskeletal pain, with the most common diagnosis being rotator cuff disease. One of the factors causing this pain is the loss of strength or endurance of the rotator cuff muscles. The muscles that are most affected include the supraspinatus and infraspinatus which allow external rotation and abduction and help to provide dynamic stabil- ity. Due to the prevalence and subsequent high cost of treating shoulder dysfunction, it is imperative to determine the most effective and efficient rehabilitation program.

Purpose: The purpose of the present study was to compare the effect of either a strength or endurance training program on the rotator cuff muscles of the shoulder.

Study Design: Randomized controlled trial

Methods: The participants were comprised of 60 healthy graduate students, with a total of 52 participants who completed the study. A 12-week training program randomly placed participants into either the control, endurance, or strength training group. Participants were then randomly assigned a treatment arm where they conducted 6 exercises that targeted mainly the posterior rotator cuff muscles. Pretesting consisted of strength assessment via a handheld dynamometer for shoulder flexion, abduction (ABD), external rotation (ER), and internal rotation (IR), while endurance was assessed using a side-lying external rotation timed en-

\footnotetext{
a Cooresponding Author:

Phil Page - Phillip.Page@FranU.edu
} 
durance test. While both training groups performed the same exercises, those placed in the strength program were instructed to complete a higher intensity (using the OMNIRES scale) and lower volume, while the endurance program was instructed to complete a lower intensity (using OMNIRES scale) and a higher volume. 3 sets of each exercise were performed, and when unable to perform them with minimal fatigue and an OMNI-RES score below the target range, they advanced to the next level of increased resistance. Compliance was tracked through weekly log sheets. Posters demonstrated the 6 exercises targeted shoulder ER and abduction.

Results: The strength training group showed an increase in ABD, ER, and IR strength. ABD showed an average increase in 2.19 pounds and ER 2.25 pounds. Since the exercises in the training program emphasized strengthening of the supraspinatus and infraspinatus, increases in ABD and ER strength were expected. IR strength showed an average increase of 3.76 pounds and while this secondary increase in IR was welcomed, it was unexpected. Increases in IR strength may have been due to an overall increase in dynamic shoulder stability gained by the exercises performed. Flexion strength did not show a significant increase. This was expected as the exercises were not specific to those muscles.

The endurance training group demonstrated a significant increase in ER endurance with an average improvement of 27.16 seconds. The endurance group showed no significant difference for all four strength measurements of the treated and untreated arm. However, while insignificant, the endurance group did yield strength gains for all four strength measurements except IR for both the treated and untreated arm.

Discussion/Conclusion: The study results were as predicted regarding both the strength and endurance training groups. Each group demonstrated increases in their perspective training modes. Based on our results from this study, these specific training protocols may be beneficial for targeting posterior shoulder muscles. The conclusion of our research raises the question of which is the best protocol regarding strength or endurance training for those with rotator cuff pathology or pain. Clinicians would benefit from future research targeting patients with rotator cuff pathology and the training program's ability to resolve and prevent injury in the long-term.

Presenting Author: Rob Manske - robert.manske@wichita.edu

\section{IMPACT OF USING AN ONLINE REHABILITATION PROGRAM FOR LOW BACK PAIN COMPARED WITH TRADITIONAL PHYSICAL THERAPY: A PILOT STUDY}

\section{Bray J}

University of St. Augustine for Health Sciences

Background: The recent Covid-19 pandemic has demonstrated the need for alternatives to face-to-face physical therapy services. Virtual physical therapy services and online rehabilitation programs are becoming more popular throughout the United States. Little research has been conducted to establish the effectiveness of these online programs.

Purpose: The purpose of this study is to investigate if an online interactive rehabilitation program improves patient pain and return to function for subjects experiencing low back pain. In addition, to investigate the outcomes of an online interactive rehabilitation program compared to traditional physical therapy.

Study Design: Case Report

Methods: This is a non-randomized clinical study of subjects that engaged in the RecoveryOne web and appbased program for recovery from low back pain compared to traditional care in the literature. Each participant was asked to complete a visual analog pain scale and an Oswestry Disability Index.

Results: The 40 subjects who completed a VAS at 4 weeks demonstrated a mean decrease of 1.275 on the visual analog scale with a p of .000 . The 25 subjects who completed an ODI at 4 weeks had a mean decrease of 3 with a p of .023. The 24 subjects who completed a VAS at 8 weeks demonstrated a mean decrease in VAS of 2 from the beginning of treatment with a $\mathrm{p}$ of .000

Discussion/Conclusion: Online rehabilitations program may be a viable alternative to face-to-face physical therapy in reducing pain and improving function in patients with non-surgical low back pain. Patients who have been diagnosed with low back pain saw a decrease in pain and increase in function after 4 weeks and an overall decrease in pain at 8 weeks utilizing an online interactive rehabilitation program that was statistically significant. An online interactive rehabilitation program may be an alternative to traditional physical therapy.

Presenting Author: Jonathan Bray - JBray@usa.edu

THE FATIGUE INDEX: AN OVERLOOKED COMPONENT OF CRITERIA FOR RETURN TO SPORT (RTS) FOLLOWING UPPER EXTREMITY INJURIES AND USING UPPER EXTREMITY FUNCTIONAL PERFORMANCE TESTS (UEFPT)

\section{Davies, GJ, Bargeron, S, Connolly, A, Hackmann, K, Lane, T, Riemann, $B L$}

Georgia Southern University Department of Rehabilitation Sciences

Purpose/hypothesis: There is a lack of evidence on the effects of fatigue on UEFPT which may influence RTS decisions. The purpose was to investigate effects of fatigue on UEFPT. We hypothesized the fatigue protocol would result in decreased performance.

Materials/methods: Forty-six participants, ages 21-34 years old (22 females) (mean age 24.7, mean weight 74.9 $\mathrm{kg}$, mean height $170.2 \mathrm{~cm}$ ) completed 3 testing sessions. First session was to establish 10-repetition maximum using seated landmine press, and weight for the fatigue protocol used $70 \%$. The operational definition of fatigue was: unable to keep up with the metronome, demonstrated compensations or lacked full range of motion for 3 reps. Sessions 2 and 3 consisted of one of the UEFPT as a pre-test, followed by fatigue protocol, finishing with same UEFPT as a posttest. Randomization determined testing sequence and 
which arm was tested. The UEFPTs were the closed-kineticchain-upper-extremity-stability-test (CKCUEST) and the seated single-arm shotput test (SSASPT).

Results: Using a paired t-test, CKCUEST demonstrated a significant decrease from pre-test to post-test $(p<0.001)$. SSASPT demonstrated significant decreases for both dominant (Dom) and non-dominant (Non-Dom) arms from pretest to post-test $(\mathrm{p}<0.001, \mathrm{p}<0.001)$.

Conclusions: Results indicated fatigue significantly reduced the CKCUEST and SSASPT post-test values supporting our hypothesis. The results suggest fatigue plays a role in decreasing performance on the UEFPT and should be considered for RTS.

Clinical relevance: If the patient performs the UEFPT in a non-fatigue state, passes, re-tests in a fatigue state and passes by not decreasing more than the MDC, we recommend discharging the patient as long as they have met all the other criteria. However, if the patient performs the UEFPT in a non-fatigue state, passes, re-tests in a fatigue state and fails it by decreasing more than the MDC, we recommend continuing rehabilitation to increase endurance and work capacity.

Presenting author: George Davies - gdavies@georgiasouthern.edu

\section{THE EFFECTS OF DRY NEEDLING ON RUNNERS: A RANDOMIZED CONTROLLED TRIAL}

\section{Dewald $M^{1,2}$, Whitten $B^{2}$, Reynolds $A^{2}$, Semprini $J^{2}$}

Affiliations: ${ }^{1}$ University of South Dakota, Department of Physical Therapy; Vermillion, SD ${ }^{2}$ Briar Cliff University, Department of Physical Therapy; Sioux City, IA.

Background: Runners are frequently plagued with injuries and are often prescribed hip strengthening as an intervention. While the efficacy of hip strengthening as an intervention is understood, less can be said about how ancillary interventions affect it. A common intervention amongst runners is dry needling. While dry needling has been shown to be effective in treating pain, less is understood about its impact on strength and function.

Purpose: The purpose of this study is to determine the effects of dry needling on hip strength and function in runners compared to sham dry needling when both are completed with a progressive resistance hip strengthening intervention.

Study Design: Randomized Controlled Trial

Methods: Ten subjects between the ages of 20-25, who routinely run, were randomized into either true dry needling and exercise group $(n=5)$ or a sham dry needling and exercise group $(n=5)$. The subjects were blinded to their intervention and the researchers were blinded to the treatments. Subjects completed a standardized progressive resistance exercise program with instruction $3 \mathrm{x}$ 's/wk for 8 weeks. Once a week subjects either received dry needling with monofilament needles or sham needling with the metal handle of the monofilament needles to the gluteus maximus, gluteus medius, tensor fascia latae, and the piriformis; supervision was provided for the progression of the hip strengthening program at this time as well. The primary outcomes collected were isometric hip extension, abduction and prone external rotation strength with a dynamometer and functional measurements with the Modified Star Excursion test.

Results: No significant difference was noted between the two groups at each of the three testing times $(p<.05)$ for strength. No significant findings were noted within either group for hip extension measurements. Significant within group difference were noted for the sham group for right hip external rotation between week 4 and week $8(\mathrm{p}=.001)$ as well as between week 1 and week $8(\mathrm{p}=.004)$. Left hip external rotation also saw significant within group findings from week 4 to week $8(p=.02)$ and week 1 to week $8(p=.01)$. There was no significance within group differences noted for hip abduction measurements.

No significance was found between the treatment and sham group with the Modified Star Excursion test. The paired samples $t$ test did show significant difference within $\mathrm{DN}$ from week 1 to 8 for left anterior, left posteromedial and left posterolateral ( $\mathrm{p}=0.036, \mathrm{p}=0.001, \mathrm{p}=0.049$ respectively). The sham group showed significant difference at all three time periods for right posteromedial $(p=0.039, p=0.009$, $\mathrm{p}=0.009$ ). Right posterolateral showed statistically significant difference at weeks $1-4(\mathrm{p}=0.036)$, while left posteromedial showed statistical significance at weeks 4-8 $(\mathrm{p}=0.026)$.

Generally, all groups and directions showed trending and significant improvements in strength and function, without significance for whether they received dry needling or sham dry needling.

Discussion/Conclusion: This study found that dry needling does not add to the intervention of hip strengthening in runners when assessed with isometric hip strength and the Modified Star Excursion test. However, the expectations and the needs of runner should be considered when deciding on intervention options beyond a simple biomechanical framework. The results of this study should be considered cautiously secondary to the low number of subjects. There is limited research regarding the effects of dry needling on strength and function in runners, more studies are needed to further investigate the potential benefits of dry needling in runners.

Presenting author: Matt Dewald - Matt.Dewald@usd.edu

\section{TREATMENT OF A PROFESSIONAL POLE}

VAULTER WITH INSERTIONAL ACHILLES

TENDINOPAHY USING TENDON FENESTRATION, SYMPTOMS AND OUTCOMES CORRELATE WITH TENDON THICKNESS AND TENDON STIFFNESS: A CASE STUDY

\section{Dewald $M$}

Affiliations: University of South Dakota, Department of Physical Therapy; Vermillion, SD

Introduction: Achilles tendinopathies are common and can occur during the peak of an athlete's career. Currently, the impact of a rehabilitation program on the biomechanical properties of Achilles tendons are poorly understood, with conflicting evidence. Tendon fenestration dry needling 
is in its infancy, with promise for chronic tendinopathies. The purpose of this case is to present a novel treatment of using tendon fenestration of the Achilles tendon, followed by a traditional tendon loading program, and the correlations of the biomechanical properties of the Achilles tendon for a professional pole vaulter with insertional Achilles tendinopathy.

Case Description: A 28-year-old professional pole vaulter with a 2-year history of left insertional Achilles tendinopathy, limiting her ability to compete, was treated initially with tendon fenestration followed by a traditional tendon loading program. The patient had pain with palpation at the Achilles insertion, pain with passive dorsiflexion and loaded plantarflexion. Her VISA-A at the evaluation was 57\%. Ultrasound imaging of the Achilles showed thickening at $8.0 \mathrm{~mm}$ vs $6.0 \mathrm{~mm}$ of the right; however, no interruptions of the internal fibrillar structure of the tendon. Tendon stiffness was measured with the MyotonPro, a myotonometer, that has been shown to be both a reliable and valid tool for tendon biomechanical measurements. Using the mean of 3 measurements, the left tendon stiffness was $733 \mathrm{~N} / \mathrm{m}$ while the right was $822 \mathrm{~N} / \mathrm{m}$, an $89 \mathrm{~N} / \mathrm{m}$ difference.

Outcomes: Over the course of 4 months the VISA-A improved 20 pts (MCID 6.5 pts), ultrasound imaging showed decreased thickness by $24 \%$ from $8.0 \mathrm{~mm}$ to $6.1 \mathrm{~mm}$, myotonometer measurements showed increased Achilles tendon stiffness from $733 \mathrm{~N} / \mathrm{m}$ to $852 \mathrm{~N} / \mathrm{m}$, a $120 \mathrm{~N} / \mathrm{m}$ increase. There was a strong positive correlation between tendon stiffness and VISA-A $(r=0.75)$. There was stronger negative correlation of tendon stiffness and tendon thickness $(r=$ -0.89 ) The patient was able to return to pain free running and jumping at this point.

Management: Tendon fenestration dry needling was completed on the first two sessions, with 10 parallel passes of dry needles towards the Achilles insertion on the calcaneal tuberosity, starting about $2 \mathrm{~cm}$ proximal. Isometrics and a heel lift were used the first two weeks. Following two weeks, a traditional tendon loading program was initiated. The tendon loading program was progressed once a week over the next 4 months, initially following the Silbernagel Protocol and then progressing using the Baxter et al. loading index to continue to incrementally load the Achilles tendon with lower extremity strengthening and plyometrics.

Discussion: This case demonstrates that tendon fenestration dry needling may be a promising addition to the treatment of chronic insertional tendinopathy. Biomechanical properties improved simultaneously as the tendon loading program progressed, while the symptoms and outcomes improved on the VISA-A. This case also demonstrated that tendon thickness can change incrementally with the progression of rehab, symptoms, and outcomes. Consistent, strict adherence to a tendon loading program with highly structured, incremental progression lead to the successful return to very high-level athletics for an international level athlete who was limited with her ability to compete for two years.

Presenting author: Matt Dewald - Matt.Dewald@usd.edu

\section{SHORT-TERM EFFECTS OF DRY NEEDLING ON HAMSTRING LENGTH: A RANDOMIZED CONTROLLED TRIAL}

\section{Dewald $M^{1,2}$, Deiter $A^{2}$, Gross $A^{2}$, Moravec $G^{2}$, Wills $D^{2}$}

Affiliations: ${ }^{1}$ University of South Dakota, Department of Physical Therapy; Vermillion, SD; ${ }^{2}$ Briar Cliff University, Department of Physical Therapy; Sioux City, IA.

Background: An impairment in hamstring length is commonly encounter in the clinic and has been linked to a variety of pathologies. While there is promising research on the impact of dry needling for pain, less is understood regarding muscle length.

Purpose: The purpose of this study is to identify the short-term effect of dry needling on hamstring length in an adult population being treated with dynamic stretching.

Study Design: Double Blinded, Randomized Controlled Trial

Methods: Twenty-three subjects volunteered with 20 subjects being included after identifying hamstring length impairments of greater than 20 degrees with the 90-90 active knee extension test. Thirty-seven hamstrings from the 20 included subjects were then randomized into a control group of dynamic stretching and sham dry needling $(n=21)$ or an intervention group of dynamic stretching and dry needling $(n=16)$. Two treatments sessions of true or sham dry needling were completed over the course of a week, in which subjects and researchers were blinded to which subjects received dry needling or sham dry needling. The dynamic exercises were completed daily with instructions and supervision from the researchers for both groups.

Results: There was a significant change in hamstring length from pre-treatment to post-treatment in both groups combined (6 degrees, $\mathrm{p}=.009$ ). However, no significance in hamstring length change between the dry needling and control groups $(\mathrm{p}=.74)$.

Discussion/Conclusion: The addition of dry needling did not alter the short-term improvements of dynamic stretching for shortened hamstring length. Based off the outcomes of this study, dynamic stretching without the addition of dry needling is sufficient to quickly improve the hamstring length of an adult with limitations. Assessing the intervention group through the lens of a case series may paint a different picture, as it would yield a significant change in hamstring length with the intervention of dry needling and dynamic exercise. Therefore, considering the context of the patient's presentation may impact the clinical decision making necessary to include dry needling with a dynamic stretching program for adults with restricted hamstring length.

Presenting author: Matt Dewald - Matt.Dewald@usd.edu

\section{ACHILLES TENDON STIFFNESS OF DIVISION 1 CROSS COUNTRY RUNNERS: IS SYMMETRY THE GOAL?}

Dewald $M$ 
Affiliations: University of South Dakota, Department of Physical Therapy; Vermillion, SD

Background: There is conflicting data on the influence of Achilles tendon (AT) stiffness on performance and injuries in athletes. Recently the assumption of symmetry has been questioned as well. For example, the greatest sprinter in history has $14 \%$ asymmetry. Should coaches and clinicians aim for symmetry or is asymmetry the norm?

Purpose: The purpose of this study is to identify if there is a relationship between AT stiffness asymmetry and performance or injuries in division 1 (D1) cross country (XC) runners.

Study Design: Case-control study

Methods: Twenty-six (20 female) D1 XC runners volunteered from a midwestern university to participate in the study at the beginning of their XC season. The means of 3 AT biomechanical measurements were collected bilaterally with the MyotonPro, a myotonometer, at the beginning of their XC season. The MyotonPro has been shown to have good to excellent intra-rater reliability and inter-rater reliability. Validity has been established against shear wave ultrasound elastography with construct validity being demonstrated as well. Per the manufacture's recommendations, the subjects were positioned prone with a bolster positioned anterior to their distal lower leg to allow a relaxed position for tendon measurements. The measurements were completed at the midline of the AT between the medial and lateral malleoli. Historical data on injuries and performances were collected with subjective history and surveys during data collection.

Results: There was no correlation in AT stiffness asymmetry and performance $(r=-.01)$. There was no difference $(\mathrm{p}=.71)$ in AT stiffness asymmetry of those with a history of injury $(n=14,4.9 \%)$ in the last year to those who were injury free $(n=12,5.8 \%)$. There was a significant difference in mean AT stiffness between the high $(726 \mathrm{~N} / \mathrm{m})$ and low $(688$ $\mathrm{N} / \mathrm{m}$ ) stiffness AT within runners $(\mathrm{p}<.01)$ with the mean AT stiffness asymmetries being $5.3 \%$.

Discussion/Conclusion: Challenging the assumptions of symmetry, there was no meaningful relationships between AT stiffness asymmetry and performance or injuries. In fact, there was $5.3 \%$ asymmetry in AT stiffness between D1 XC runners included in this study. Asymmetry may have evolved, developed, or been trained to its current state with good reason. Aiming to control asymmetry of a single or a few variables without a comprehensive understanding of its impact may be a shortsighted practice.

Presenting author: Matt Dewald - Matt.Dewald@usd.edu

\section{ACHILLES TENDON STIFFNESS OF FEMALE DIVISION 1 CROSS COUNTRY RUNNERS WHO USE ORAL CONTRACEPTIVES}

\section{Dewald M, Luken E, Peters D, Polzin J, Thum J, Gustaf S}

Affiliation: University of South Dakota, Department of Physical Therapy; Vermillion, SD

Background: Previous tendon research does not provide data on the Achilles tendon (AT) stiffness of Division 1 (D1) female cross country (XC) runners who use oral contracep- tives (OC). Limited information is known about the effect of estrogen and progesterone on tendon stiffness in D1 XC athletes, with conflicting presumptions being that there is a decrease in tendon stiffness based off less inclusive data. A decrease in AT stiffness may have an impact on injury and performance in D1 female XC runners, therefore an investigation on the impact of OC is warranted. With the findings of decreased AT stiffness in athletes who use OC, coaches and clinicians may prophylactically prescribe tendon loading programs to minimize the potentially negative effects of decreased stiffness.

Purpose: The purpose of this study is to identify if there is a difference in AT stiffness among female D1 XC athletes who use OC and do not.

Study Design: Case-control study

Methods: Twenty current D1 female XC runners volunteered from a midwestern university to participate in the study. Six of the 20 subjects use OC and 14 do not use OC. AT biomechanical measurements were collected bilaterally with the MyotonPro, a myotonometer, at the beginning of their XC season. The MyotonPro has been shown to have good to excellent intra-rater reliability and interrater reliability. Validity has been established against shear wave ultrasound elastography with construct validity being demonstrated as well. Per the manufacture's recommendations, the subjects were positioned prone with a bolster positioned anterior to their distal lower leg to allow a relaxed position for tendon measurements. The measurements were completed at the midline of the AT between the medial and lateral malleoli. The average of 6 measurements, 3 per tendon, were used to calculate the mean AT stiffness of each athlete.

Results: The mean AT stiffness of the OC users measured $664 \mathrm{~N} / \mathrm{m}$, while the nonusers measured $702 \mathrm{~N} / \mathrm{m}$. The results of an independent t-test indicate significantly lower AT stiffness of D1 female XC runners that use OC $(\mathrm{M}=664$, $\mathrm{SD}=23.35)$ than those who do not $(\mathrm{M}=702, \mathrm{SD}=48.5)$, $\mathrm{t}(18)=-2.138, \mathrm{p}=.033$.

Discussion/Conclusion: Female D1 XC runners who use OC have significantly less AT stiffness. Coaches and clinicians should consider a prophylactic AT loading program to minimize potentially adverse effects of OC use. Future research should examine how a prophylactic AT loading program impacts the AT stiffness, performance, and risk of injury.

\section{Presenting author: Matt Dewald - Matt.De wald@usd.edu}

\section{THE EFFECTS OF VERBAL CUES ON QUADRICEPS EMG ACTIVITY DURING A QUADRICEPS SETTING EXERCISE}

Frerichs, $C^{1}$, DeHope, $J^{2}$, Chamberlain, $M^{3}$, Bassett, $D^{4}$, Farmer, $B^{1}$, Kyvelidou, $A^{1}$, Magrini, $M^{5}$, Grindstaff, $T L^{1}$

${ }^{1}$ Department of Physical Therapy, Creighton University, Omaha, NE, USA; ${ }^{2}$ Physical Therapist, Covenant Health, Crossville, TN, USA; ${ }^{3}$ Physical Therapist, HonorHealth, Scottsdale, AZ, USA; ${ }^{4}$ Physical Therapist, F.I.T. Muscle \& Joint Clinic, Kansas City, MO, USA; ${ }^{5}$ Department of Exercise 
Science and Pre-Health Professions, Creighton University, Omaha, NE, USA

PURPOSE: A quadriceps setting (quad set) exercise is commonly utilized following knee injury, but there is great variation in clinical prescription. The purpose of this study was to determine if internal, external, or visual cues results in the greatest quadriceps electromyographical (EMG) activity during a quadriceps setting exercise.

METHODS: Thirty healthy individuals (15 males, 15 females; age $=25 \pm 2.5 \mathrm{y}$, mass $=79.3 \pm 14.2 \mathrm{~kg}$, height $=176.9 \pm 9.5$ $\mathrm{cm})$ volunteered for this study. Peak EMG amplitude of the vastus lateralis was determined during each exercise condition. Participants performed the quadriceps setting exercise and were given one of five cues in a randomized order: internal cue "tighten your thigh muscles," internal cue "push your knee down," external cue "push into the bolster," external cue "push into the strap," or visual biofeedback (mTrigger) using the cue "raise the value on the screen as high as you can." A repeated measures ANOVA and associated post-hoc tests, with corrected alpha levels ( $p<0.005)$, were used to determine differences in normalized EMG activity between conditions.

RESULTS: There was a significant difference between conditions $(\mathrm{p}<.001)$. Post-hoc comparisons indicated both visual biofeedback $(83.2 \pm 24.9 \%)$ and "press into the strap" (76.8 $\pm 24.4 \%)$ produced significantly greater $(\mathrm{p}<0.001)$ EMG activity than the push knee down $(53.2 \pm 27.0 \%)$, tighten thigh $(52.7 \pm 27.3 \%)$, or push into the bolster $(50.8 \pm 26.3 \%)$ conditions. There was no significant difference $(p=0.10)$ between the visual biofeedback and "press into the strap" conditions as well as no significant difference ( $p>0.38)$ between the push knee down, tighten thigh, or push into the bolster conditions

CONCLUSION/SIGNIFICANCE: Visual biofeedback and pressing into the strap produced the greatest EMG activity compared to the other internal and external cues. If the clinical aim during a quadriceps setting exercise is to obtain the greatest volitional muscle recruitment, the use of visual biofeedback or pressing into a strap is recommended.

Presenting Author: Connor Frerichs cfr50377@creighton.edu

\section{THE EFFECTS OF CERVICAL SPINE POSITION ON SHOULDER ROTATION STRENGTH}

\section{Giordano $K,{ }^{1}$ Oliver $G^{2}$}

${ }^{1}$ Creighton University Department of Physical Therapy, Phoenix, AZ, ${ }^{2}$ Auburn University School of Kinesiology, Auburn, AL

Background: Shoulder rotation strength testing is commonly used in clinical examinations of the shoulder. Populations prone to shoulder injury, such as overhead athletes and manual trades workers, place their shoulders under tremendous amounts of stress when their cervical spine is in non-neutral positions. Given standard clinical assessments test strength with a neutral cervical spine, investigation into the effects of cervical spine rotation is warranted.

Purpose: The purpose of this study was to determine the effects of a rotated cervical spine on isokinetic shoulder internal and external rotation strength.
Study Design: This was a repeated measures, within subject, crossover study.

Methods: Fifty-two healthy individuals $(170 \pm 10 \mathrm{~cm}$, $73 \pm 18 \mathrm{~kg}$ ) participated. Concentric shoulder internal and external rotation strength were tested through a $90^{\circ}$ arc on an isokinetic dynamometer with the shoulder elevated $90^{\circ}$ in the frontal plane and $45^{\circ}$ anterior to the frontal plane (scapular plane). Tests were performed with the participant's cervical spine in neutral in both planes, maximally rotated contralaterally in the frontal plane, and maximally rotated ipsilaterally with the shoulder in the scapular plane. Testing order was randomized. Data were imported into MATLAB for statistical parametric mapping analysis.

Results: Rotating the cervical spine contralaterally with the shoulder in the frontal plane resulted in a significant decrease in external $(p<0.001)$ and internal $(p=0.023)$ rotation strength with the forearm within $15-20^{\circ}$ of vertical. Rotating the cervical spine ipsilaterally with the shoulder in the scapular plane resulted in a significant decrease in external rotation strength $(\mathrm{p}<0.001)$ throughout nearly the entirety of the motion with peaks roughly around $20^{\circ}$ and $60^{\circ}$ from the horizontal, and internal rotation strength $(\mathrm{p}<0.001)$ the last $60^{\circ}$ towards the horizontal.

Discussion/Conclusion: About $35 \%$ of patients with common neuromusculoskeletal shoulder pathologies have cervical involvement. Because populations like overhead athletes and trades workers require use of their shoulders in altered cervical spine positions, there is reason to believe cervical spine position may be responsible for the strength differences seen in this study.

Presenting Author: Kevin Giordano -
kag0070@auburn.edu

\section{PRETIBIAL STRENGTH AND GAIT CHARACTERISTICS IN INDIVIDUALS WITH UNILATERAL PLANTAR FASCIITIS VERSUS HEALTHY CONTROLS}

\section{Granado, $M^{1}$, Lohman, $E^{2}$, Gordon, $K^{3}$, Daher, $N^{4}$ \\ 1 Department of Physical Therapy Education, Western University of Health Sciences, Pomona, CA, USA; ${ }^{2}$ Depart- ment of Physical Therapy, Loma Linda University, Loma Linda, CA, USA; ${ }^{3}$ Physical Therapy and Human Movement Sciences, Northwestern University, Chicago, IL, USA; ${ }^{4}$ School of Allied Health Professions, Loma Linda Univer- sity, Loma Linda, CA, USA}

Background: While higher ground reaction forces (GRF) have been a suspected cause of plantar fasciitis (PF), its relationship is still unclear. Muscle fatigue is known to cause higher GRFs, but no known studies have examined the relationship between pretibial strength and PF.

Purpose: The purpose of this study is to compare pretibial strength and specific gait characteristics in those with unilateral PF with gender-matched healthy participants.

Study Design: Case-control study.

Methods: Twenty participants with unilateral PF (mean age 47 years, 13 females) were compared between involved and uninvolved feet as well as with twenty gender-matched healthy controls (mean age 43 years, 13 females). A handheld dynamometer was used to measure the strength of tib- 
ialis anterior (TA) and extensor hallucis longus (EHL). A pressure treadmill was utilized to quantify gait parameters such as initial toe contact and max GRF.

Results: Some evidence demonstrated EHL strength to be weakened in involved feet of the PF group when compared to healthy controls $(0.07 \mathrm{~kg}(0.02)$ vs $0.08 \mathrm{~kg}(0.03)$, $\mathrm{P}=0.11, \mathrm{\eta} 2=0.4)$. As well, the involved side of the $\mathrm{PF}$ group exhibited some evidence of early toe initial contact versus healthy controls (34.6\% gait cycle (10.6) vs $38.8 \%$ gait cycle (13.3), $\mathrm{P}=0.14, \eta 2=0.4)$. Mean max GRF was also found to be significantly higher in the PF group versus healthy controls (involved versus controls, $780.4 \mathrm{~N}$ (98.6) vs $700.2 \mathrm{~N}$ (126.9) $\mathrm{P}=0.02$, $\mathrm{\eta} 2=0.7$; uninvolved vs controls, $789.8 \mathrm{~N}$ (100.0) vs 692.7 N (126.0) $\mathrm{P}=0.01, \eta 2=0.9$ ).

Discussion/Conclusion: While no significant differences were noted with TA strength between groups, some evidence for a weak EHL coinciding with early initial toe contact during gait may exist in those with PF. This study is relevant as it possibly identifies a muscle group needing to be strengthened when treating PF.

Presenting author: Michael Granado
mgranado@westernu.edu

\section{TREATMENT OF UNRESOLVED SEVER'S DISEASE LEADING TO POSSIBLE ACHILLES' TENDINOPATHY IN A 15-YEAR-OLD MALE: A CASE REPORT}

\section{Grant L, Brantley S, Swiggum M \\ Wingate University}

Introduction: Calcaneal apophysitis (Sever's Disease) is one of the most common causes of adolescent heel pain, affecting 3.7 per 1000 . This condition is believed to be an overuse injury that occurs due to repetitive submaximal loading and resulting microtraumas on the growth plate caused by traction of the Achilles tendon. There is currently no gold standard for diagnosis or treatment for Sever's Disease.

Case Description: This retrospective case study evaluated the effectiveness of physical therapy treatment on a 15-year-old male who was diagnosed with Sever's Disease at age 11 by a physician. The goal of intervention was to reduce the patient's pain and allow for him to return to age-appropriate recreational physical activity. The subject ceased participation in track and field when initially diagnosed due to his pain. Daily bilateral heel pain, particularly during running, jumping, and quick cutting motions were still experienced when physical therapy interventions were initiated.

Clinical Impression: The patient presented with left $>$ right heel pain on average of $6 / 10$ on the visual analog pain scale that was worse in the mornings once he got out of bed when he bore weight through the lower extremities. He reported pain levels increased to a 9/10 during activities including pushing off the leg while scootering or while walking or jumping. Pain symptoms were occurring daily, sometimes multiple times, and episodic pain would last from 1-5 minutes. At the time of the initial evaluation, the patient demonstrated bilateral weakness with both knee flexion and extension, as well as hypomobility at bilateral talocrural joints. Patient was also tender to palpation bilaterally at mid-portion of Achilles tendons, greater than the insertional point of Achilles on the calcaneus. Treatment interventions included lower extremity strengthening, passive and active stretching, balance and manual therapies including stretches, PNF, and soft tissue techniques. Strengthening included both closed and open chain exercises as well as concentric and eccentric exercises.

Outcomes: At conclusion of treatment, the patient's pain levels decreased from at worst $9 / 10$, to 3/10. Duration of symptoms decreased from 1-5 minutes to 30 seconds-3 minutes. Lower leg pain occurrence was reduced from daily to only with activity. Patient's morning heel pain was completely alleviated. The patient also had improved pain free range of motion, improved strength in all planes of the bilateral lower extremity and improved muscle flexibility as demonstrated by muscle length measurements.

Discussion: This case study demonstrated that physical therapy intervention can reduce pain severity and frequency in patients with long standing Sever's Disease. Our episode of treatment resulted in decreased pain and improved function during both activities of daily living and leisure activities. Sever's Disease is one of the most common diagnoses of young athletes and a common source of pain. It is reported that $16 \%$ of emergency department visits involving active children are due to posterior heel pain. At the time of this study there are no current gold standard diagnostic outcome measures or recommendations for treatment of Sever's disease. The absence of these tools could lead to long-term effects being experienced for these patients including not returning to sport and long-term health implications. Long-term effects could also lead to psychosocial issues for these adolescents. Sever's disease is assumed to be a self-limiting disease that resolves with activity modification and will completely resolve by the time the child's development slows down. This study supports the need for further research of this disease to treat patients that do not recover based on the traditional treatments and expected recovery time.

Presenting Author: Lisa Grant - 1.grant@wingate.edu

\section{THE ROLE OF CORE BODY TEMPERATURE ON LACTATE PRODUCTION}

\section{Heller $C^{1}$, Grahn $D^{1}$, Cao $V^{1}$, Choi $A^{2}$ \\ ${ }^{1}$ Stanford University, ${ }^{2}$ Choi Sports Rehab}

Background: Blood lactate studies indicate a close association between lactate threshold and fatigue. That observation led to the common concept that fatigue was caused by a build-up of lactate when individuals exceeded their aerobic capacity. The concept of lactate production causing fatigue has been disproven. Rather than lactate causing fatigue, we propose that mechanisms of fatigue result in lactate production. One of those fatigue mechanisms is limitation of ATP production in response to hyperthermia. This adaptation might be due to the inactivation of pyruvate kinase at high temperatures. If that hypothesis is correct, lactate threshold should also be temperature sensitive.

Purpose: We evaluated effects of core body temperature on lactate threshold and production of lactate. The hypoth- 
esis was that rise in core body temperature hastens fatigue during exercise, and a manifestation of this fatigue mechanism is a rise in lactate production.

Study Design: crossover paired tests

Methods: Two studies were performed. In study one, 12 college age subjects ( 3 females, 9 males) performed incremental graded exercise on a treadmill (SciFit). Core body temperatures $\left(\mathrm{T}_{\mathrm{es}}\right)$ were measured with self-inserted esophageal thermocouples (Mallinckrodt Medical). Each subject completed two tests, one starting at a $\mathrm{T}_{\text {es }}$ of $37^{\circ} \mathrm{C}$ and one starting at a $\mathrm{T}_{\mathrm{es}}$ of $38^{\circ} \mathrm{C}$, with the order of the two tests randomized. Starting $\mathrm{T}_{\mathrm{es}}$ was achieved by resting in a $45^{\circ} \mathrm{C}$ environment until the target $\mathrm{T}_{\text {es }}$ was reached. Speed of the treadmill was $3.5 \mathrm{mph}$ and $0 \%$ incline, then raised $2 \%$ every 3 minutes until volitional stop. Blood lactate (Nova Biomedical) was measured at every change in workload. Lactate threshold was determined as the workload when blood lactate reached $4.0 \mathrm{mmol} / \mathrm{L}$. We hypothesized that the $38^{\circ} \mathrm{C}$ tests would reach lactate threshold sooner and at a lower workload. In cross-over study two, 14 subjects ( 5 females, 9 males) randomly completed three trials. In one trial, the subjects, dressed in summer workout attire, simply rested at room temperature for 60 minutes. In trial two, dressed in summer attire, they rested at $45^{\circ} \mathrm{C}$ for 60 minutes. In trial three, subjects dressed in Personal Protective Equipment (PPE) rested at $45^{\circ} \mathrm{C}$ for 60 minutes. All subjects performed a five minute treadmill walk at $60 \%$ of their calculated maximal workload at the end of the 60 minute rest period. We hypothesized that only individuals with a higher starting core temperature would show a rise in blood lactate levels post-intervention.

Results: In the first study, the $38^{\circ} \mathrm{C}$ group reached lactate threshold with an average of 220 Watts of work, while the $37^{\circ} \mathrm{C}$ group reached lactate threshold with an average of 263 Watts. This 43 Watts difference was significant $(\mathrm{p}<0.001)$. In the second study, subjects in trials 1 and 2 showed no increase in $\mathrm{T}_{\mathrm{es}}$ or blood lactate at the end of the rest period or treadmill challenge. In trial 3, subjects showed an increase in $\mathrm{T}_{\mathrm{es}}$ to $38.8^{\circ} \mathrm{C}$ at the end of the rest, but no increase in blood lactate. However, after the treadmill challenge, the subjects in trial 3 showed a more than doubling of blood lactate $(\mathrm{p}<0.001)$. The lactate response was only seen in response to a mild exercise challenge in hyperthermic subjects, but hyperthermia alone did not affect their blood lactate.

Discussion/Conclusion: A state of elevated core temperature impairs the ability of subjects to respond to exercise challenges without a significant rise in blood lactate. Effective management of core body temperature may prolong sustainable efforts in training and competition before the body reaches lactate threshold.

$$
\text { Presenting author: Andy Choi }
$$
achoi94402@gmail.com

\section{TREATMENT OF PLANTAR FASCIITIS USING DRY NEEDLING: A CASE REPORT}

Hortz B, Falsone $S$

A.T. Still University, Athletic Training
Introduction: Plantar heel pain (PHP) is a common problem causing pain in the foot under the heel and can eventually affect both foot function and gait. Plantar fasciitis is commonly treated conservatively with joint and soft tissue mobilization, self-stretching, foot orthoses, and night splinting. Dry needling has demonstrated to be an effective method of treating plantar fasciitis in multiple RCTs and has been reported to be beneficial in managing residual pain and dysfunction. Still, there is limited published literature on dry needling a myofascial sequence as part of the treatment of plantar fasciitis.

Case Description: The subject was a 38-year-old female runner referred by a podiatrist for evaluation and treatment of plantar fasciitis, including dry needling and therapy for persistent pain in the right foot. She was treated four times over three weeks in addition to education in an independent home exercise program. During the initial examination, she reported her resting pain as 60/100 (100 mm visual analog scale) and first-step pain in am 82/100. The patient scored a $41 / 104$ on the FADI subscale and a 5/32 on the FADI Sports subscale. A Fascial Manipulation ${ }^{\circledR}$-Stecco method ${ }^{\circledR}$ patient history and evaluation were completed. This evaluation revealed dense and painful CCs in the myofascial sequence of retromotion and extrarotation at many segments of the posterior chain, including the retro-coxa and ex-coxa at the hip, retro-genu, ex-genu at the knee, and retro-talus in the lower leg. These findings indicated a myofascial sequence that may be a factor in her condition extending from the hip to the foot. She had had a pes planus foot bilaterally and tenderness to palpation at the origin medial calcaneus with some mild tenderness along the length of the central band of the PF. Goniometry revealed that she had 2 degrees of dorsiflexion with the knee extended and 5 degrees with the knee flexed, demonstrating ROM restriction but a negative sign of gastrocnemius equinus, a negative Silverskiold test. Knee ROM was WNL bilaterally, and Hip ROM was WNL bilaterally except internal rotation (24 degrees R, 32 degrees L) and external rotation (28 degrees $R, 45$ degrees $L$ ). She had a negative Tinel test along the tibial nerve's distal portion, helping to rule out tarsal tunnel syndrome. Given the case presentation, the clinician felt the treatment of the plantar fasciitis diagnosis should include treatment beyond the local foot pain, incorporating the posterior chain and particularly the hip where history, the fascial evaluation, and ROM restrictions demonstrated possible involvement.

Outcome: The dry needling intervention was beyond the local plantar fascia and incorporated 11 locations from the foot up the posterior chain with the addition of two electric stim channels. The patient had reduced pain as measured by a VAS (60 to 15), increased function as measured by the functional ankle disability index (41 to 88), and dorsiflexion increases at the ankle (2 to $10 \mathrm{deg}$ ).

Discussion: In this case, this patient had previously done several rounds of prescribed physical therapy. We believe the inclusion of dry needling of the hip and the associated exercises for the hip was novel for the patient and beneficial. Dry needling targeted the dense and painful CC's in the posterior chain to change the densification of these myofascial centers of coordination. To the authors' knowledge, this is the first article that demonstrates that needling these 
CC's may indeed work to decrease densification, decreasing pain, and increasing function.

Conclusion: This case illustrates the use of local and lower quarter dry needling in addition to a home exercise program to provide a favorable outcome in a patient with plantar fasciitis.

Presenting author: Brian Hortz - hortzb@gmail.com

\section{DOES HIGH MEDIAL ELBOW STRESS DURING PITCHING COMPROMISE THE DYNAMIC STABILIZERS OF THE ELBOW?}

\section{Mullaney MJ, McHugh MP \\ Nicholas Institute of Sports Medicine and Athletic Trauma}

Background: The flexor carpi ulnaris (FCU) and flexor digitorum superficialis (FDS) are thought to provide dynamic stability to the medial elbow, with a lesser contribution from the pronator teres (PT).

Purpose: The purpose of this study was to determine if baseball pitchers with higher valgus elbow torque (an index of medial elbow stress) experience greater postgame FCU and FDS fatigue and slower subsequent recovery.

Study Design: Descriptive laboratory/field study.

Methods: A pilot study was performed to identify valid tests of FCU and FDS Function. Surface EMG signals were recorded from the FCU, FDS and PT during hand-held dynamometry testing of middle finger (MF) and ring finger

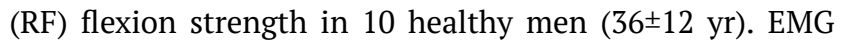
amplitudes, expressed as percent of maximal voluntary contraction (MVC) based on standard MVC tests for each muscle, were compared between tests and muscles with repeated measures analysis of variance (ANOVA).

Field Testing was performed in NCAA D3 baseball pitchers during the Fall season and Spring preseason. MF, RF and grip strength were tested prior to, immediately after, and one day after 14 pitching performances. Elbow valgus torque was measured from an inertial measurement unit, housed in a compression sleeve, worn on the elbow during pregame bullpen pitches (removed prior to game). Pitchers were categorized as having high or low valgus torque (> or < $50 \mathrm{Nm} ; 62 \pm 7 \mathrm{Nm}$ vs $32 \pm 3 \mathrm{Nm}$ ). Effect of valgus elbow torque on fatigue and strength recovery was assessed using mixedmodel ANOVA.

Results: Pilot Study: MF force was greater than RF force $(75 \pm 17 \mathrm{~N}$ vs. $52 \pm 9 \mathrm{~N}, \mathrm{P}<0.001)$. For the MF test EMG activations were $74 \%$ FDS, 66\% FCU and 35\% PT (muscle effect $\mathrm{P}=0.032$ ). For the RF test activations were 93\% FCU, 61\% FDS and 23\% PT (muscle effect $\mathrm{P}=0.005$ ).

Field Testing: Baseline MF force was greater than RF force $(77 \pm 11 \mathrm{~N}$ vs. $58 \pm 11 \mathrm{~N}, \mathrm{P}<0.001)$ and neither were different between pitchers with high $(n=8)$ versus low $(n=6)$ valgus torque $(\mathrm{P}=0.288, \mathrm{P}=0.541)$. Pitchers threw $58 \pm 12$ pitches with no difference between pitchers with high versus low valgus torque $(\mathrm{P}=0.263)$. Pitchers with high valgus torque $(n=8)$ experience marked post-game MF fatigue $(88 \%$ of baseline) and incomplete recovery the following day (95\%), while pitchers with low valgus torque $(n=6)$ exhibited no strength changes (107\% of baseline post game, $106 \%$ a day later; group $\mathrm{x}$ time $\mathrm{P}=0.022)$. Similarly, pitchers with high valgus torque experience post-game RF fatigue (94\% of baseline) with minimal recovery the following day (96\%), while pitchers with low valgus torque exhibited no fatigue (114\% of baseline post game) and no delayed strength loss (107\% a day later; group $\mathrm{x}$ time $\mathrm{P}=0.048$ ). By contrast, grip strength was decreased post game ( $95 \%$ of baseline) and had not recovered by the following day (95\%; time effect $\mathrm{P}=0.013$ ) but these effects were not different between pitchers with high versus low valgus torque $(\mathrm{P}=0.143)$. Valgus torque explained $40 \%$ of the variance in post-game RF fatigue $(\mathrm{P}=0.015)$. A combination of valgus torque and pitch count explained $57 \%$ of the variance in post-game MF fatigue $(\mathrm{P}=0.010)$. Neither valgus torque $(\mathrm{P}=0.129)$ nor pitch count $(\mathrm{P}=0.845)$ were related to post-game grip strength.

Conclusions: Based on EMG analyses, the MF and RF strength tests provided a functional assessment of the dynamic stabilizers of the medial elbow. Based on field testing, high valgus torque at the elbow during pitching results in greater fatigue and slower recovery of the dynamic stabilizers of the medial elbow. These are the first data to show a link between elbow stress during pitching and compromised dynamic stability in the medial elbow.

Presenting Author: Mike Mullaney laneypt@gmail.com

\section{THE ROLE OF SPINAL MANIPULATION AS A MODERATOR OF CERVICAL SPINE POSITION ON SHOULDER ROTATION STRENGTH}

\section{Oliver $G,{ }^{1}$ Giordano $K^{2}$}

${ }^{1}$ Auburn University School of Kinesiology, Auburn, AL; ${ }^{2}$ Creighton University Department of Physical Therapy, Phoenix, AZ

Background: Emerging data shows that altering the cervical spine's position many negatively impact shoulder rotation strength. This may be problematic in populations that require strenuous use of their shoulders. Because spinal manipulations result in acute increases in cervical spine range of motion and extremity strength, there is reason to believe spinal manipulation may mitigate the deleterious effects of a rotated cervical spine on shoulder rotation strength.

Purpose: To assess spinal manipulation as a moderator of cervical spine rotation on shoulder rotation strength.

Study Design: This was a randomized control trial.

Methods: Fifty-one participants $(170 \pm 10 \mathrm{~cm}, 73 \pm 18 \mathrm{~kg})$ underwent concentric shoulder internal and external rotation strength testing on an isokinetic dynamometer. The shoulder was tested through a $90^{\circ}$ arc at $60^{\circ} / \mathrm{s}$ with the shoulder elevated $90^{\circ}$ in the frontal and $45^{\circ}$ anterior to the frontal plane (scapular plane). Tests were performed with the participant's cervical spine in neutral, maximally rotated contralaterally in the frontal plane, and maximally rotated ipsilaterally with the shoulder in the scapular plane. Testing order was randomized. Participants received either cervicothoracic spinal manipulation or a sham manipulation then were tested with the same protocol following, and 30 minutes following treatment. Multilevel regression models were used to compare peak torque. 
Results: In both the frontal and scapular plane, the level 2 explanatory model was superior to the model including a group*time*position interaction, indicating spinal manipulation did not positively affect shoulder internal or external rotation strength. Time consistently had a negative coefficient, indicating both groups decreased strength over time.

Discussion/Conclusion: Current data do not suggest a thrust manipulation mitigates the effects of an altered cervical spine position on shoulder rotation strength. However, when identical models were run splitting the groups by joint cavitation rather than manipulation, models including the group*time* cavitation interaction were superior to level 2 explanatory models, with interaction estimates being positive. This provides weak evidence that cavitations may play a role in moderating shoulder rotation strength, but further research is needed.

Presenting Author: Gretchen Oliver - goliver@auburn.edu

\section{CLINICAL INFERENTIAL STATISTICS: MAKING CLINICAL DECISIONS WITH ESTIMATION STATISTICS}

\section{Page, $P$}

Franciscan University DPT Program, Baton Rouge LA

Evidence-based practice (EBP) encourages clinicians to make clinical decisions based on the 'best-available' evidence. Clinical inference is the process of applying knowledge (best evidence) prior to action in the context of a clinical situation such as an individual patient treatment. Clinicians sometimes have difficulty in identifying the 'best' evidence, particularly when faced with assessing and interpreting research statistics. Statistical inference imparts results of a study sample on a represented population, while clinical inference applies results of a study on individual patients.

Statistical inference relies on several assumptions (eg, sample size, variance, distribution, homogeneity) to infer sample results on clinical populations; unfortunately, some clinical studies used in making clinical decisions fail to meet these assumptions. To compound this problem, the commonly reported "p-value" for statistical significance is of little value in clinical decision making, although most clinicians (and researchers) are unaware of this. While the p-value provides the probability of incorrectly rejecting the null hypothesis as a dichotomous "Yes-No" statistical decision, it does not necessarily provide the evidence needed to make an informed clinical decision.

Clinicians can use estimation statistics derived from representative samples to determine the clinical significance of research outcomes, thus supporting evidence-informed decision making. Estimation statistics can help clinicians identify the magnitude and direction of an outcome (effect size), range of likely outcomes in a population (confidence interval), and the best estimate of the true value of the outcome (point estimate). Clinicians should use the minimal clinically important difference (MCID) values to determine if a study's particular outcome measure is clinically meaningful. These statistics can then be used to make a clinical inference in the context of the research outcome and the individual patient.

Presenting

Phillip.Page@FranU.edu

\section{EVALUATION OF MUSCULOSKELETAL CLINICAL PRACTICE GUIDELINES FOR RECOMMENDED THERAPEUTIC INTERVENTIONS}

\section{Page P, Mistretta C, Thompson J, Brittain K}

Franciscan University DPT Program, Baton Rouge LA

Background: Clinical practice guidelines (CPGs) are designed to provide clinicians with recommendations for evidence-based interventions. Although these resources are available, research indicates many PTs fail to choose evidence-based guidelines during treatment, likely due to lack of time, resources, and generalized results. While there are many physical therapy CPGs in publication by various organizations around the world, CPGs may be of different levels of quality or difficult for clinicians to interpret.

Purpose: The purpose of this systematic review was to evaluate the quality of musculoskeletal CPGs from the Academy of Orthopedic Physical Therapy and develop a decision matrix for choosing evidence-based physical therapy interventions.

Methods: MEDLINE, PEDro, and SPORTDiscus databases were searched, while PTNow was also cross-referenced in the search. Articles were included if they meet the following criteria: musculoskeletal clinical practice guidelines developed by the AOPT within the past 10 years. Exclusion criteria included CPGs from other authors/organizations, non-musculoskeletal guidelines, and guidelines that did not use the AGREE II quality assessment tool. Data were extracted and developed into a matrix that includes musculoskeletal conditions, recommended interventions, and level of evidence for the interventions.

Results: The most-recommended interventions were therapeutic exercise and manual therapy, respectively. While many recommendations were given, few were of high-quality evidence.

Conclusion: Having access to evidence supporting optimal treatments or identifying non-beneficial treatments is important for clinicians in making decisions to guide patient treatment.

\begin{tabular}{l}
$\begin{array}{c}\text { Presenting author: } \\
\text { Phillip.Page@FranU.edu }\end{array}$ \\
\hline
\end{tabular}

\section{OSTEOCHONDRAL AND LIGAMENT INJURY PATTERNS IN ANKLE FRACTURES: A MULTI- CENTER STUDY}

\footnotetext{
Carreira $D^{1}$, Shaw $D^{1}$, Harris $T^{2}$, McCullough $K^{3}$, Kreulen $C$ ${ }^{4}$, Martin $R^{5}$, Acevedo ${ }^{6}$

${ }^{1}$ Peachtree Orthopedics, Atlanta, GA; ${ }^{2}$ University of California Los Angeles, Los Angeles, CA; ${ }^{3}$ Orthopaedic and Sports Medicine Clinic of Kansas City, Kansas City, MO; ${ }^{4}$ University of California Davis, Davis, CA; ${ }^{5}$ Duquesne University, Pittsburgh, PA; ${ }^{6}$ Southeast Orthopedic Specialists, Jacksonville, FL
} 
Background: When treating ankle fractures, the presence of talar osteochondral lesions and the extent of ligamentous injury is associated with the severity of injury. The Danis-Weber classification system has been used to predict potential ligament damage.

Purpose: The purpose of this study is to validate the ligament injury patterns that have been proposed to occur with the Danis-Weber Classification and identify the cartilage injury pattern that occurs on the talus with each fracture type.

Study Design: Retrospective Cohort Study

Methods: A prospective, multi-center foot and ankle arthroscopy registry was queried for patients who underwent arthroscopic treatment of ankle fractures from 2017 to 2020. Preoperative and intraoperative findings were noted, including the Danis-Weber fracture classification, presence and location of osteochondral damage, and unstable deltoid, and/or syndesmotic ligaments. Kendall's tau-b, a nonparametric correlation coefficient for ordinal variables, was used to measure the strength and direction of association between Weber fracture type and the presence of a syndesmotic injury, deltoid ligament tear, medial malleolus fracture, or osteochondral lesion. The locations of osteochondral damage were compared as percentages.

Results: 73 subjects were prospectively collected as part of a multicenter ankle arthroscopy database. Average subject age was 43 years (SD = 17) with $59 \%$ female and $41 \%$ male. A significant association between the presence of a syndesmotic injury and fracture type was identified, with syndesmotic injuries more likely occurring with a Weber $\mathrm{C}$ fracture $\left(73 \%, \mathrm{r}_{\mathrm{T}}=0.44, \mathrm{p}<0.0005\right)$. A significant association was not identified between Weber classification and a deltoid tear $\left(23 \%, \mathrm{r}_{\mathrm{T}}=0.74, \mathrm{p}=0.47\right)$ or medial malleolus fracture $\left(32 \%, \mathrm{r}_{\mathrm{T}}=-0.001, \mathrm{p}=0.79\right)$. Location of the talar osteochondral lesions were as follows: $22 \%$ medial-anterior, $22 \%$ lateral-anterior, 22\% lateral-central, $17 \%$ central-anterior, 9\% medial-central, 4\% later-posterior, $4 \%$ central. There was no significant association identified between Weber classification and the location of the osteochondral injury ( $\mathrm{p}=0.99)$.

Discussion/Conclusion: Concurrent injuries with syndesmotic disruption, deltoid ligament tears, medial malleolus fractures, and osteochondral lesions have been proposed to be associated with Weber $\mathrm{C}$ ankle fractures. However, in this multicenter study, only syndesmotic injuries were associated with the Weber $\mathrm{C}$ classification. This study found similar rates of medial malleolus fractures and osteochondral lesions in Weber B and C type fractures.

Presenting author: RobRoy Martin - martinr280@duq.edu
HAMSTRING STRAIN INJURY: CLINICAL PRACTICE GUIDELINES LINKED TO THE INTERNATIONAL CLASSIFICATION OF FUNCTIONING, DISABILITY, AND HEALTH FROM THE ACADEMY OF ORTHOPAEDIC PHYSICAL THERAPY AND AMERICAN ACADEMY OF SPORTS PHYSICAL THERAPY OF THE AMERICAN
PHYSICAL THERAPY ASSOCIATION

Martin RL, ${ }^{1}$ Cibulka MT, ${ }^{2}$ Bolgla LK, ${ }^{3}$ Koc $T,{ }^{4}$ Loudon $J K,{ }^{5}$ Manske RC, ${ }^{6}$ Weiss L, ${ }^{7}$ Christoforetti JJ, ${ }^{8}$ Heiderscheit $B C^{9}$

${ }^{1}$ Duquesne University, Pittsburgh, PA; ${ }^{2}$ Maryville University, St. Louis, MO; ${ }^{3}$ Augusta University, Augusta, GA; ${ }^{4}$ Kean University, Union, NJ; ${ }^{5}$ Rockhurst University, Kansas City, MO; ${ }^{6}$ Wichita State University, Wichita, KS; ${ }^{7}$ New York Football Giants, East Rutherford, NJ; ${ }^{8}$ Texas Health Sports Medicine, Frisco, TX; ${ }^{9}$ University of Wisconsin-Madison, Madison, WI

Background: Hamstring strain injuries (HSI) are common in activities that involve high-speed running, jumping, kicking, and/or explosive lower extremity movements with rapid changes in direction, including lifting objects from the ground. Sports such as track, soccer, Australian Rules football, American football, and rugby have the highest frequency of reported injuries. HSI may result in considerable impairment, activity limitation, and participation restriction, including time lost from competitive sports. In professional sports, a HSI may be associated with significant financial costs.

Purpose: The Academy of Orthopaedic Physical Therapy of the American Physical Therapy Association has an ongoing effort to create evidence-based clinical practice guidelines (CPGs). The aims of this review were to provide a concise summary of the contemporary evidence and to develop recommendations to promote evidence-based practice.

Study Design: A systematic review

Methods: A literature review was performed from 1967 to June 2021. Individual clinical research articles were graded to support specific for diagnosis, examination, injury prevention, interventions, re-injury risk, and return to play guidelines (RTP).

Results:

Diagnosis: Clinicians may diagnose a HSI when an individual presents with a sudden onset of posterior thigh pain during activity, that is reproduced pain when the hamstring is activated and/or stretched, associated muscle tenderness with palpation and loss of function. (Weak evidence)

Examination: Clinicians should quantify knee flexor strength following HSI by using either a HHD or isokinetic dynamometer. (Strong Evidence)

Clinicians should assess hamstring length measuring knee extension deficit with the hip flexed to $90^{\circ}$ using an inclinometer. (Strong Evidence)

Clinicians may measure the length of muscle tenderness and location from the ischial tuberosity to assist in predicting timing of RTP. (Weak Evidence)

Clinicians may assess for increased anterior pelvic tilt and abnormal trunk and pelvic control during functional movements. (Expert Opinion) 
Clinicians may include objective measures of an individual's ability to walk, run, and sprint when documenting changes in activity and participation over the course of treatment. (Weak Evidence)

Clinicians should use the Functional Assessment Scale for Acute Hamstring Injuries (FASH) to document changes from before and after interventions. (Moderate Evidence)

Injury Prevention Program: Clinicians should include the Nordic hamstring exercise with other components of warm-up, stretching, stability training, strengthening, and functional movements (sport-specific, agility and highspeed running). (Strong Evidence)

Intervention: Clinicians should use hamstring muscle eccentric training, added to stretching, strengthening, stabilization, and progressive running programs, to improve RTP time after a HSI. (Moderate Evidence)

Clinicians should incorporate progressive agility and trunk stabilization, added to a comprehensive impairmentbased treatment program with stretching, strengthening, and functional exercises, to reduce re-injury after a HSI. (Moderate Evidence)

Clinicians may use soft-tissue mobilization, nerve glides, and therapeutic modalities to assist in the healing process and shorten the period of disability after a HSI. (Expert Opinion)

Re-injury and Return to Play: Clinicians should consider a previous HSI a risk factor for a future re-injury, with those having sustained a more recent injury being at higher risk. (Moderate Evidence)

Clinicians should consider the absence of an appropriately progressed, comprehensive impairment-based functional exercise program a risk factor for re-injury and programs that do not specifically include eccentric training a risk factor for re-injury as well as delayed RTP. (Moderate Evidence)

Clinicians should use hamstring strength, pain level at the time of injury, number of days from injury to pain-free walking, and area of tenderness measured at initial evaluation to estimate time to RTP. (Moderate Evidence)

Discussion/Conclusion: This CPG supports evidencebased physical therapy practice in the diagnosis, examination, injury prevention, intervention after injury, and risk assessment for re-injury and return to play decisions for those with a HSI.

Presenting author: RobRoy Martin - martinr280@duq.edu

\section{THE CONCURRENT VALIDITY OF STRENGTH MEASUREMENTS FOR NECK FLEXION AND EXTENSION OBTAINED BY HAND-HELD DYNAMOMETRY. A COMPARISON WITH THE MULTI-CERVICAL UNIT.}

Hangartner $M^{1}$, Bühler $S^{1}$, Romano $F^{2}$, Agostino $D^{2}$, Feddermann-Demont $N^{2}$, Bizzini $M^{2,3}$

1 ZHAW Zurich University of Applied Sciences, Winterthur, Switzerland

2 Swiss Concussion Center, Schulthess Clinic, Zürich, Switzerland
3 Human Performance Lab, Schulthess Clinic, Zürich, Switzerland

Background: While rehabilitation and prevention of sports-related concussions have become a field of interest in physiotherapy research, so far, the relevance of neck strength in association with concussion remains unclear. Valid devices and user-friendly measuring protocols for clinical practice are needed to further investigate the importance of neck strength in rehabilitation.

Purpose: The purpose of the present study was to evaluate the concurrent validity of a user-friendly and time-efficient protocol to measure cervical flexion and extension strength using a hand-held dynamometer (HHD) and the Multi-Cervical Unit (MCU) as a reference device.

Study Design: Descriptive Laboratory Study

Methods: The MCU and an HHD measured neck flexion and extension strength of 30 active, healthy males (mean age 27.1 years) on one measuring day. Data analysis used maximum voluntary strength values in Newton $(\mathrm{N})$. Concurrent validity was determined using paired t-test and Pearson correlation. Bland-Altman plots and boxplots were used to illustrate differences between the devices.

Results: Neck flexion and extension strength were significantly different between the devices $(p<0.01)$. Weaker correlations between the two devices were found for flexion ( $r=0.35$, 95\% CI: -0.02 to 0.63 to $0.8, p<0.06$ ) than for extension ( $r=0.63,95 \% \mathrm{CI}: 0.35$ to $0.81, \mathrm{p}<0.001)$. Bland-Altman Plots revealed sizable limits of agreement for both directions.

Conclusion: Neck strength measured with an HHD and the protocol used were different from those obtained with the MCU. The strength of the tester and deviations in positioning potentially limited absolute agreement between the MCU and the HHD. Therefore, values retrieved through different devices should be interpreted with caution and not used interchangeably by clinicians. Future studies should focus on establishing a gold standard for neck strength measurements.

Presenting author: Ashley Campbell - ashleymariecampbell@gmail.com

\section{ACUTE ISOLATED LATERAL RETINACULAR TEAR EVALUATION, TREATMENT, AND OUTCOME: A CASE REPORT}

Chris Gallina, PT, DPT, CSCS, Russ Paine, PT, Walt Lowe M.D.

Background: An isolated tear of the lateral retinaculum is a rare and unreported diagnosis within the literature. Guidelines for this condition both conservatively and surgically are essentially non-existent resulting in a difficult route to determination of treatment and outcomes. Both medial and lateral patellar stability are critical when returning to function and sporting activities. The dilemma of this case was to determine what symptoms might be resolved through conservative management and when should surgical intervention occur? The description of this case provides a history of the treatments and outcomes of this unusual injury.

Case Description: A 15 year old female athlete sustained an injury during a basketball game when she landed 
from receiving a rebound (1/19/2020). The patient immediately felt a "pop" in her right knee. Immediately after the injury the patient developed an unusual bruising pattern isolated to the medial and lateral aspects of the skin surrounding the patella. Initial X-Ray and MRI evaluation of the injury revealed an undetermined diagnosis with no apparent structural damage. Initial management included bracing the knee in full extension for 1 month followed by the ability to perform range of motion activities without restriction. Due to initial restrictions in range of motion the patient developed significant impairments including range of motion deficits (0-20 degrees flexion), complete quadriceps inhibition, and severe muscle splinting. Three months following initial injury, the patient received a second physician opinion resulting in a diagnosis of an isolated retinacular tear (4/20/2021).

Preoperative Outcomes: Following the diagnosis, initial interventions were implemented to restore normal range of motion and improve quadriceps inhibition. Range of motion was initially addressed using dual channel biofeedback on the quadriceps and hamstrings to relax the antagonistic muscle groups when utilizing muscle energy techniques and manual stretching. These techniques restored full range of motion $0-150$ within 2 weeks. Quad deficits were addressed using biofeedback training in both the open and closed chain positions in order to provide feedback for muscle activation. Preoperatively the patient was able to restore $60 \%$ of quad function. Balance, gait, and hip stability was also addressed to improve lower extremity function. After completing conservative rehabilitation, the patient was able to return to basic functional activities (walking and low level strengthening). Unfortunately, she was unable to perform higher level activities such as jogging, cutting, jumping. Interestingly, following multiple at- tempts at these high level movements, the patient would develop a similar bruising pattern along the area of the lateral retinaculum and developed symptoms of pain and instability. Because of these symptoms the decision to undergo surgical repair of the lateral retinaculum was made on $8 / 27 / 2021$ with the goal of returning to play sports.

Postoperative Outcomes: Following surgery the patient immediately experienced improved patellar tracking and quadriceps activation, as well as decreased pain and swelling. The patient also underwent a comprehensive course of physical therapy and strength and conditioning resulting in full range of motion, symmetrical lower extremity strength ( $0 \%$ quad deficit), and minimal pain. These outcomes led to the ability to run, jump, cut, and ultimately return to sport within 4 months post surgery without further complications.

Discussion: Not all knee injury evaluations are simple and straightforward, with some requiring high level experienced clinicians working as a team to solve unusual cases. We found that early normalization of strength and range of motion is imperative for progression to functional lower extremity activities with biofeedback being a very useful technique for assisting in this process. We also know patellar stability is vital for performance of high level athletic activities and through this case, determined that surgical intervention proved necessary for return to sport following an isolated retinacular tear.

Presenting author: Chris Gallina cgallin2@mail.naz.edu

Submitted: October 10, 2021 CDT, Accepted: October 15, 2021 CDT 